\section{OWE-027 EFFECTS OF PRIOR JEJUNAL FEEDING ON GASTRIC EMPTYING AND SYMPTOMS IN PATIENTS WITH DIABETIC GASTROPARESIS}

${ }^{1,2}$ Mark Fox* ${ }^{2,3}$ Caroline Hoad, ${ }^{2}$ Emily Tucker, ${ }^{2}$ Johnathan White, ${ }^{2,3}$ Luca Marciani, ${ }^{2}$ Helen Parker. ${ }^{1}$ University of Zürich, Zurich, Switzerland; ${ }^{2}$ NIHR Nottingham Digestive Diseases Centre, Nottingham University Hospitals NHS Trust and University of Nottingham, Nottingham, UK; ${ }^{3}$ Sir Peter Mansfield Imaging Centre, School of Physics and Astronomy, University of Nottingham, Nottingham, UK

\subsection{6/gutjnl-2018-BSGAbstracts.415}

Introduction Diabetic gastroparesis (DG) affects up to 20\% patients with type I Diabetes Mellitus (DM). Impaired gastric function is thought to be the cause of nausea, vomiting, abdominal pain and impaired glycaemic control. DG does not respond reliably to intensive insulin regimes or prokinetic medications. Jejunal nutrition (JN) is an option in patients that cannot maintain weight. The benefits are thought to be improved nutrition and glycaemia; however, we have observed that some DG patients can eat normally during JN.

We propose that DG represents a failure of oral nutrition to 'switch' the stomach from the fasted to the fed state with nutrition delivered direct to the jejunum triggering neuro-hormonal mechanisms that induces normal gastric function. This study tests the hypothesis that $\mathrm{JN}$ prior to a meal improves postprandial symptoms) and gastric function.

Methods Diabetic patients with severe symptoms (GCSI >27), diabetic controls (GCSI <14) and healthy controls (HC) entered a randomised, double blind, controlled trial. Glycemia was controlled. NJ feeding tube was placed. Liquid nutrient ( $2 \mathrm{kcal} / \mathrm{min}$ ) or water was infused for $60 \mathrm{~min}$. The validated Nottingham Test Meal was then ingested (NTM liquid: $400 \mathrm{~mL}, 300 \mathrm{kcal}$; solid: 12 non-nutrient agar beads). Symptoms were documented (VAS), gastric function by MRI and the GI-peptide response was monitored. Mixed model analysis compared response to intervention and between groups

Results 9 DG patients, 9 diabetic and 12 HC were recruited. There was no difference in demographic features between groups. DG patients reported higher satiety, bloating and pain after NTM ingestion than diabetic and healthy controls $(\mathrm{p}<0.05)$.

Sensations were not affected by JN in controls; however, fullness, bloating and pain were reduced by JN in DG patients $(\mathrm{p}<0.01$, figure).

Liquid GE was similar in both study arms $(\mathrm{p} \geq 0.727)$. Antral contraction wave (ACW) frequency was $2.7 / \mathrm{min}$ in health and was highest in diabetic controls $(3.1 / \mathrm{min})$. Solid GE was more rapid after JN than water and, again, was highest in diabetic controls ( 2 ( 1 to 3 ) and 3 (1 to 7$)$ more beads emptied@60 min, both p<0.05). Compared to water, JN induced a greater GI-peptide response; however, this was less pronounced in both diabetic groups. A correlation with symptoms or GE was not identified.

Conclusions This RCT demonstrates beneficial effects of prior JN on fullness, bloating and pain after a $400 \mathrm{~mL}$ test meal in DG patients. Additionally, solid GE was accelerated after JN; however, this effect was not limited to DG patients. These findings suggest that effects of JN on symptoms are not mediated by improvement in GE but on other aspects of gastric function or visceral sensitivity. Future studies will identify patients likely to benefit from this novel approach to treatment.
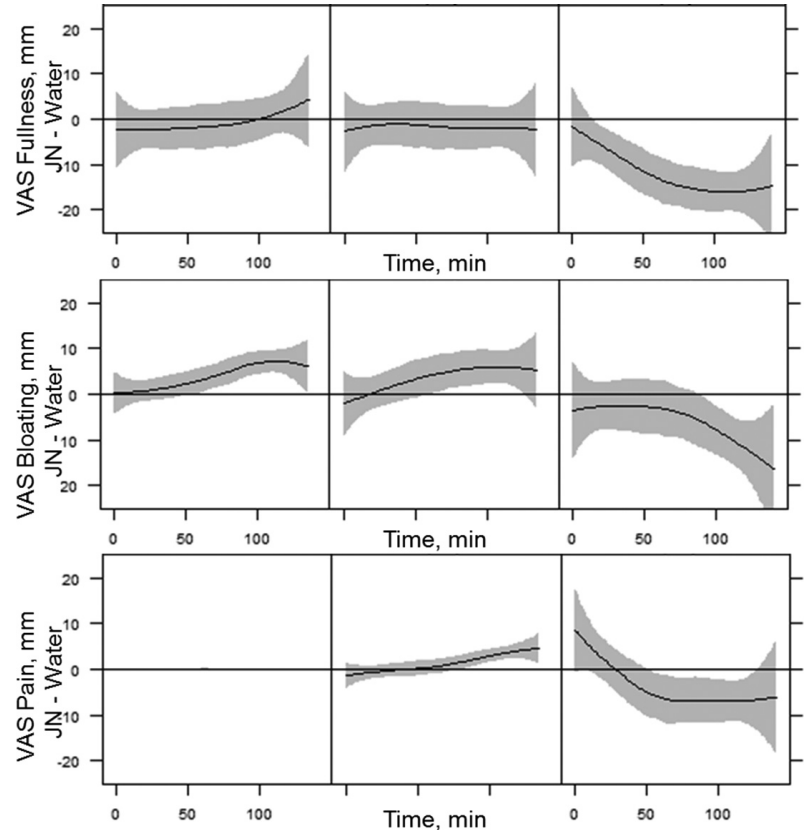

Abstract OWE-027 Figure 1

Average sensation showing difference between JN and placebo (water) study arms with 95\% confidence bands. Negative values demonstrate a reduction in symptoms with $\mathrm{JN}$

\section{OWE-028 ACTIB TRIAL (ASSESSING COGNITIVE BEHAVIOURAL THERAPY IN IRRITABLE BOWEL): A MULTICENTRE RANDOMISED CONTROLLED TRIAL}

${ }^{1}$ Prof Hazel Everitt*, ${ }^{2}$ Sabine Landau, ${ }^{1}$ Paul Little, ${ }^{1}$ Prof Felicity Bishop, ${ }^{1}$ Gillian O'Reilly, ${ }^{2}$ Alice Sibelli, ${ }^{2}$ Rachel Holland, 'Stephanie Hughes, ${ }^{2}$ Sula Wingassen, ${ }^{2}$ Paul McCrone, ${ }^{2}$ Kim Goldsmith, ${ }^{3}$ Nicholas Coleman, ${ }^{4}$ Robert Logan, ${ }^{2}$ Trudie Chlader, ${ }^{2}$ Rona Moss-Morris. ${ }^{1}$ University of Southampton, Southampton, UK; ${ }^{2}$ Kings College, London; ${ }^{3}$ Southampton University Hospital, Southampton; ${ }^{4}$ Kings College Hospital, London

\subsection{6/gutjnl-2018-BSGAbstracts.416}

Introduction Irritable Bowel Syndrome (IBS) is a common chronic gastrointestinal condition, characterised by abdominal pain, bloating and change in bowel habit. Medications have limited benefit and many patients experience ongoing symptoms with significant impact on quality of life. Cognitive behavioural therapy (CBT) for IBS is recommended in NICE guidelines but NHS access is very limited. Objective: To determine the clinical effectiveness of therapist telephone delivered CBT and web-based CBT self-management with minimal therapist support compared to treatment as usual in adults with refractory IBS.

Methods ACTIB is a National institute for Health Research (NIHR) multicentre randomised controlled trial. Participants: Adults ( $\geq 18$ years) with refractory IBS who had been offered first-line therapies (eg, antispasmodics, antidepressants or fibrebased medications) and had continuing IBS symptoms $\geq 12$ months, were recruited over 23 months from primary and secondary care in the south of England and London. The interventions were therapist telephone delivered CBT (TCBT) or Web-based CBT self-management with minimal therapist support (WCBT) versus treatment as usual (TAU). Main 
outcome measures: IBS Symptom Severity Score (IBS SSS) and Work and Social Adjustment Scale (WSAS). Baseline and follow up data was patient reported and collected on-line at 3 , 6 and 12 months. Analysis: Intention-to-treat with multiple imputation at 12 months.

Results 558/1452 (38.4\%) patients screened for eligibility recruited: 186 randomised to TCBT, 185 WCBT, 187 TAU. Mean baseline IBS SSS 265.0. At 12 months TAU IBS SSS score was 205.6, compared to 61.6 points lower for TCBT $(95 \%$ CI $89.5 ; 33.8 ; \mathrm{p}<0.001)$ and 35.5 lower for WCBT (95\% CI 58.0;13.1; $\mathrm{p} \geq 0.002)$. WSAS score: TAU $=10.8$ at 12 months and 3.5 lower with TCBT (95\% CI 5.1; 1.9; $\mathrm{p}<0.001)$, 3.0 points lower with WCBT (95\% CI 4.6; 1.3; $\mathrm{p}<0.001)$. Secondary outcomes: Subjects Global improvement of symptoms (SGA) $84.8 \%$ responders TCBT at 12 months compared to $41.7 \%$ TAU OR $6.1 \quad(95 \%$ CI $2.5 ; 15.0$; $\mathrm{p}<0.001)$ and $75.0 \%$ for WCBT OR 3.5 (95\% CI 2.0 to 6.1 ; $\mathrm{p}<0.001$ ). Patient enablement (PEQ) $78.3 \%$ responders TCBT, $23.5 \%$ TAU OR $9.2(95 \%$ CI $4.3 ; 19.4 ; \mathrm{p}<0.001)$ and $54.8 \%$ for WCBT OR 3.6 (95\% CI $2.1 ; 6.1$; p $<0.001)$.

Conclusions To date, this is the largest trial of CBT for IBS worldwide. Both CBT arms showed significant improvements in IBS outcomes compared to TAU, sustained at 12 months. TCBT had larger effects than WCBT. CBT for IBS can be effectively delivered to a broad range of patients with refractory IBS.

\section{OWE-029 MAGNETO-ELECTRIC STIMULATION OF THE HUMAN CEREBELLUM PREVENTS SWALLOWING DYSFUNCTION INDUCED BY A CORTICAL VIRTUAL LESION} ${ }^{1}$ University Of Manchester, Manchester, UK; ${ }^{2}$ Salford Royal Hospital, Salford, UK

\subsection{6/gutjnl-2018-BSGAbstracts.417}

Introduction Repetitive transcranial magnetic stimulation (rTMS) is a neurostimulatory technique which can be used to alter neuronal activity within targeted regions of the brain. Furthermore, in post stroke dysphagia, recovery of swallowing function is thought to be related to increased activity in the undamaged cortical swallowing hemisphere. ${ }^{1}$ Here, we wanted to determine if stimulation of the cerebellum, known to be activated during swallowing ${ }^{2}$ can enhance swallowing when disrupted by a virtual lesion as a prelude to using cerebellar stimulation therapeutically.

Aim To compare the effects of ipsilateral and contralateral $10 \mathrm{~Hz}$ cerebellar rTMS versus sham stimulation on swallowing behaviour following a virtual lesion to the pharyngeal motor cortex.

Method Healthy human participants $(n \geq 10)$ were intubated with a pharyngeal catheter. Baseline swallowing performance was then measured using a water swallowing reaction time task. Participants received $10 \mathrm{~min}$ of $1 \mathrm{~Hz}$ rTMS (virtual lesion) to the pharyngeal motor cortex which elicited the largest pharyngeal motor evoked potentials. This causes a disruption of swallowing behaviour. Over 3 separate visits to the laboratory, participants were then randomised to receive 250 pulses of $10 \mathrm{~Hz}$ cerebellar rTMS to the ipsilateral side, contralateral side or sham (2). Swallowing performance was measured at $15 \mathrm{~min}$ intervals up to an hour after cerebellar rTMS. Results rTMS was well tolerated by all subjects. Sham stimulation was associated with the expected increase in fast swallow time latency $\left(\chi^{2} \geq 11.429 p \geq 0.044\right)$ with changes from baseline at 15 min post intervention ( $\mathrm{Z} \geq$ - Abstract OWE-029 Figure 1

1.988, $\mathrm{p} \geq 0.047)$ and $60 \mathrm{~min}$ post intervention $(\mathrm{Z} \geq-1.988$, $\mathrm{p} \geq 0.047)$ and poorer performance in challenged swallows at $30 \mathrm{~min}$ post intervention $(\mathrm{Z} \geq-2.352, \mathrm{p} \geq 0.019)$. By contrast, participants who received ipsilateral or contralateral cerebellar rTMS showed no evidence for the expected disruption of fast and challenge swallows compared to baseline ( $p>0.05)$, implying a reversal effect (Fig 1).

Conclusion Ipsilateral and contralateral $10 \mathrm{~Hz}$ cerebellar rTMS was able to block the disruptive effects of a virtual lesion in the swallowing motor cortex. There were no differences ipsilateral and contralateral cerebellar stimulation on swallowing behaviour, suggesting that activation of either cerebellar hemisphere might be therapeutically useful in dysphagic stroke.

\section{REFERENCES}

1. Hamdy $\mathrm{S}$, et al. Recovery of swallowing after dysphagic stroke relates to functional reorganization in the intact motor cortex. Gastro 1998

2. Vasant, et al. High-frequency focal repetitive cerebellar stimulation induces prolonged increases in human pharyngeal motor cortex excitability. J Physiol 2015

\section{OWE-030 A DOSE RANGING STUDY OF TRANS-SPINAL MAGNETIC STIMULATION FOR THE TREATMENT OF FAECAL INCONTINENCE}

${ }^{1}$ Shaheen Hamdy ${ }^{*},{ }^{2}$ Xuelian Xiang, ${ }^{2}$ Amol Sharma, ${ }^{2}$ Tanisa Patcharatrakul, ${ }^{2}$ Rachael Parr, ${ }^{2}$ Patricia Hall, ${ }^{1}$ Ali Abukardugha, ${ }^{2}$ Satish Rao. ' University of Manchester, Salford, UK; ${ }^{2}$ Augusta University, Augusta, USA

\subsection{6/gutjnl-2018-BSGAbstracts.418}

Introduction Current treatments for faecal incontinence (FI) are only modestly effective. FI is characterised by significant anorectal neuropathy, yet treatments for neuropathic FI are limited. In a randomised dose ranging trial, we investigated the plausibility and optimal frequency of a novel neuromodulation therapy by administering repetitive translumbar (rTLMS) and transsacral magnetic stimulation (rTSMS) in patients with FI

Methods FI patients ( $\geq 1$ episode/week) were randomised to receive weekly rTLMS and rTSMS treatments with either $1 \mathrm{~Hz}, 5 \mathrm{~Hz}$, or $15 \mathrm{~Hz}$, over six weeks. Two trains of 300 stimulations each were given at 4 sites (Total $\geq 2400$ pulses), by applying transcutaneous magnetic stimulation via a focal coil to the lumbar and sacral regions. Daily FI episodes and bowel symptoms were assessed with prospective stool diaries and compared before and after treatment. FI severity index (FISI) and subject's global assessment (SGA) were also compared. Patients with $\geq 50 \%$ decrease in weekly FI episodes were considered responders.

Results Twenty-six FI patients, $\mathrm{F} / \mathrm{M} \geq 18 / 8$ participated; 9 were randomised to $1 \mathrm{~Hz}, 8$ to $5 \mathrm{~Hz}$ and 9 to $15 \mathrm{~Hz}$ respectively. Results summarised in Table 1 . The weekly FI episodes decreased significantly in the $1 \mathrm{~Hz} \quad(\mathrm{p} \geq 0.004)$ and $15 \mathrm{~Hz}$ group $(p \geq 0.023)$, but not in $5 \mathrm{~Hz}$ group $(p \geq 0.281)$ when compared to baseline, but there was no difference between groups $(p=0.170)$. There was a significant difference between responder rates $(p=0.024)$ with the $1 \mathrm{~Hz}$ group showing a significantly higher responder rate $(88.9 \%)$ than the $5 \mathrm{~Hz}$ group (25\%), but not between other groups. After treatment, the FISI score increased by $34.6 \% \pm 18.4 \%$ in $1 \mathrm{~Hz}$ group, $12.0 \% \pm 4.9 \%$ in $5 \mathrm{~Hz}$ group, and $17.6 \% \pm 16.1 \%$ in $15 \mathrm{~Hz}$ group, but there was no difference between groups ( $\mathrm{p} \geq 0.652)$. Complete or considerable improvement in FI symptoms was reported by $66.7 \%$ in $1 \mathrm{~Hz}$ group, $37.5 \%$ in $5 \mathrm{~Hz}$ group and 